\title{
SATISFACCIÓN LABORAL EN AUXILIARES DE ENFERMERÍA
}

\author{
JOB SATISFACTION IN NURSING ASSISTANTS
}

\author{
MARTHA ISABEL \\ LASSO-QUINTERO \\ Fundación Universitaria de \\ Popayán, Colombia. \\ *Correspondencia: \\ martha.lassoldocente.fup.edu.co
}

\author{
JUAN JAVIER \\ VESGA-RODRÍGUEZ \\ Universidad El Bosque, \\ Colombia. \\ Ph.D. \\ juanjaviervesgalagmail.com \\ ORCID: 0000-0003-2382-5104
}

\author{
JULIO CESAR OSSA \\ Fundación Universitaria de \\ Popayán, Colombia. \\ juceossadgmail.com \\ ORCID: 0000-0002-3079-3318
}

\author{
ANGELA PATRICIA \\ GIRON OJEDA \\ Fundación Universitaria de \\ Popayán, Colombia. \\ angela.gironadocente.fup.edu.co \\ ORCID: 0000-0003-4620-2305
}

Este artículo analiza el nivel de satisfacción en el personal Auxiliar de Enfermería en dos servicios de una E.S.E de la ciudad de Popayán-Colombia. La investigación fue de carácter descriptivo transversal, con un enfoque cuantitativo. Participaron 60 Auxiliares de enfermería. Se utilizó el cuestionario Font Roja, la información se analizó por medio del programa estadístico SPSS (v25) para el nivel de satisfacción y se calculó el test de Anova de un Factor para diferencias significativas a. En términos generales, los Auxiliares de Enfermería de la E.S.E se ubican en un rango medio de satisfacción en los factores evaluados. Al desagregar el nivel de satisfacción laboral por factores sociodemográficos solo se encontró diferencias en las comparaciones por servicio: mayor nivel de satisfacción en el área de Urgencias que en la de Neonatos. Estadísticamente en relación al género las diferencias no fueron significativas, pero a nivel descriptivo se reveló que los hombres se encuentran más satisfechos que las mujeres. Los resultados sugieren mayor observación y estrategias de intervención que coadyuven a elevar el nivel de satisfacción en los trabajadores.

Palabras clave: Satisfacción en el trabajo, auxiliar de enfermería, motivación, políticas de salud.
This article analyzes the level of satisfaction of auxiliary nursing personnel in two services of an E.S.E. in the city of PopayánColombia. The research was of a descriptive transversal nature, with a quantitative approach. Sixty nursing assistants participated. The Font Roja questionnaire was used, the information was analyzed by means of the statistical program SPSS (v25) for the level of satisfaction and the one-factor Anova test was calculated for significant differences. In general terms, the Nursing Assistants of the E.S.E. are located in the medium range of satisfaction in the factors evaluated. When disaggregating the level of job satisfaction by sociodemographic factors, differences were only found in the comparisons by service: higher level of satisfaction in the Emergency area than in the Neonatal area. Statistically in relation to gender the differences were not significant, but at a descriptive level it was revealed that men were more satisfied than women. The results suggest further observation and intervention strategies to help raise the level of worker satisfaction.

Keywords: Job satisfaction, nursing assistant, motivation, health policies. 


\section{INTRODUCCIÓN}

Las organizaciones sanitarias fueron unas de las más afectadas con la incursión del Covid-19. Las intensas demandas en salud alteraron las dinámicas del servicio prestado y el cumplimiento de los estándares de calidad. Especialmente, el personal de enfermería fue sometido a diversos retos que intensificaron los ritmos de trabajo, el esfuerzo físico y emocional (Dantas et al., 2020; Oliva -Yarlaque y Chávarry-Ysla, 2021) vulnerando las posibilidades de bienestar en el trabajo. Las organizaciones dependen en gran medida del desempeño de sus trabajadores para satisfacer las necesidades de la sociedad en general, y la Satisfacción Laboral (SL) es piedra angular para dicho propósito (Madero- Gómez, 2020).

Abordar la SL contribuye en la comprensión de los procesos que se relacionan con la productividad, el desempeño (Avendaño et al., 2021; Chiang y Ojeda, 2013; Vargas et al., 2018) y el bienestar del trabajador (Cardoza et al, 2019; Pujol-Cols y Foutel, 2019). Temas de gran interés para las organizaciones y personas en general. El trabajo es fuente indispensable para la sobre y supervivencia. Allí se experimenta todo tipo de sensaciones interpretadas como positivas o negativas. Al consumir la mayor parte del tiempo productivo de las personas, es principal en la construcción de identidades, significados, bienestar social y material.

La SL ha sido estudiada fundamentalmente desde dos perspectivas. De acuerdo con la revisión literaria realizada por Pujol-Cols y Dabos (2018), la primera implica una concepción instrumental que aborda variables como absentismo, rotación, compromiso y desempeño; la segunda, propone la satisfacción como un determinante del bienestar físico y psicológico. En esta se distingue, aunque aún incipiente, el compromiso moral de las ciencias sociales con el estudio de la relación ser humano - trabajo en función de un mejor vivir.

La SL se refiere a la visión general que tiene un individuo frente a la organización. Se trata del afecto y valoración positiva de un empleado hacia su trabajo (Griffin et al., 2017; Li et al., 2020; Pedraza, 2018). Sin embargo, no se debe minimizar a una respuesta emocional lvariabilidad entre una emoción positiva o negatival puesto que comprende fenómenos de mayor complejidad derivados de la dimensión subjetiva de cada individuo como las percepciones (Spector, 1997). Estos procesos cognitivos y evaluativos juegan un papel crucial en la disposición de conductas en el escenario laboral (Orejuela, 2014; Robbins y Judge, 2017).

Diversas investigaciones revelan hallazgos que explican el nivel de SL asociado a diversos factores. Newstrom (2011) y Vásquez et al. (2021) encontraron relaciones significativas entre las variables sociodemográficas ledad, el nivel ocupacional y el tamaño de la organización) y el nivel de satisfacción. Otros autores mencionan factores relacionados con el salario, la contratación, el número de horas trabajadas (Cifuentes-Rodríguez y ManriqueAbril, 2014; Ruiz, 2019) y las características del trabajo (Pedraza, 2018) e incluso se profundiza en el papel de la personalidad y las disposiciones psicológicas (De la Iglesia et al., 2019; Pujol-Cols, (2019); Salessi y Omar, 2018). El estudio de estos aspectos ha cobrado importancia en el campo de la Psicología Organizacional y del Trabajo (POT), con especial interés en profesionales de la salud, dadas sus condiciones laborales de sobrecarga de tareas, déficit de tiempo, jornadas laborales largas e intensas (Ansoleaga y Ahumada, 2020; Blanch, 2014; Gálvez-Hernando et al., 2019).

Los factores mencionados traen consigo una serie 
de sucesos que impactan el sistema de salud, especialmente a los prestadores del servicio vinculados a contextos asistenciales. La naturaleza de su trabajo los somete a situaciones causantes de estrés laboral, afectando su salud mental (Veliz-Burgos et al., 2018). El personal de enfermería, conexo entre el médico y el usuario, lleva consigo la mayor carga en las tareas del cuidado del paciente (Orcasita y Ovalle, 2019). En este sentido, investigaciones en Latinoamérica sostienen que, factores asociados con la carga laboral, salario y turnos, son precursores de insatisfacción laboral en enfermeras (Troya et al., 2020). El género es una variable que suma a tal situación, pues las enfermeras, además de sus actividades profesionales, deben suplir las exigencias que demanda el hogar y, ello conduce a niveles de satisfacción media (Da Penha et al., 2019; Plascencia et al., 2016), indicador que se mantiene (medio) al ser comparados con el personal médico, quienes presentan mayores niveles de SL (Holguín y Contreras, 2019). Otros estudios, en el área de enfermería, han encontrado relación entre niveles de satisfacción y factores de riesgo psicosocial (Rivera-Rojas et al, 2020), afectaciones en la salud mental como el burnout (Carlos, 2020), y, con emociones positivas como la felicidad (Duche y Rivera, 2019).

Profundizar sobre la SLen el personal de enfermería cobra mayor sentido en el contexto de emergencia sanitaria que vive el mundo en la actualidad. Este es considerado parte esencial en la lucha contra la pandemia por el rol de cuidado permanente que prestan a los usuarios, lo cual los exponen a mayores desafíos. Así que, por un lado, proteger su bienestar es una acción crucial para la respuesta acertada y eficiente ante la emergencia de salud pública, y, por otro, para garantizar la calidad en la prestación del servicio de salud (Cook et al, 2021).
Este estudio tiene como propósito analizar el nivel de SL del personal auxiliar de enfermería en dos unidades de servicios al interior de una Empresa Social del Estado (E.S.E) de la ciudad de Popayán Colombia. De manera particular, se analizan variables sociodemográficas, variables externas e internas de la organización para ser discutidas a la luz de los niveles de $S L$ que pueden influir en el personal asistencial con propósitos de preservación de la salud y vida de los pacientes.

\section{MÉTODO}

\section{Diseño}

A través de un estudio comparativo de corte transversal y siguiendo una estrategia asociativa (Ato et al., 2013) se analiza la relación de variables examinando las diferencias que existen entre dos grupos de auxiliares de enfermería al interior de las unidades de Urgencias y Neonatos de una E.S.E. en la ciudad de Popayán.

\section{Instrumento}

Se empleó el cuestionario Font Roja (Aranaz, 1988); se usó la versión validada para Colombia (Manrique-Abril et al., 2019) conformado por 24 ítems. Las respuestas se obtienen a partir de una escala Likert con un rango de puntuación de 1 a 5 que responde al nivel de frecuencia, siendo 1 siempre y 5 nunca. La SL es medida a través de 9 factores: satisfacción por el trabajo, tensiones relacionadas con el trabajo, competencia profesional, presión del trabajo, promoción profesional, relación interpersonal con los jefes/as, relación interpersonal con los compañeros, características extrínsecas del estatus y monotonía laboral. 
En el análisis de los datos se utilizó el paquete estadístico SPSS versión 25. Para la identificación del nivel de satisfacción se hizo, en primer lugar, análisis descriptivo que permitió conocer el nivel de satisfacción $y$, en segunda instancia, el análisis de varianza (Anova de un factor). La normalidad de la muestra, se verificó a través de la prueba de Shapiro Willk (López-Roldán y Fachelli, 2016) para grupos relacionados, cuya comparación es simultánea (Hernández et al., 2014).

\section{Participantes}

Un total de 60 auxiliares de enfermería participaron en este estudio, adscritos a dos servicios (30 urgencias y 30 área de neonatos). El Universo fue de 470 enfermeros(as) auxiliares de la E.S.E. de Popayán. La selección de la muestra fue no probabilística por conveniencia. Los criterios de inclusión fueron la voluntad manifiesta de participar en la investigación (diligenciamiento y firma del consentimiento informadol y contar con un tiempo de contratación superior a 6 meses.

\section{Procedimiento de análisis de datos}

El análisis de los datos se obtuvo con la aplicación de la prueba ANOVA de un factor, que permitió comparar el comportamiento de la variable satisfacción en diferentes grupos poblacionales letarios, género, estado civil, servicio y antigüedad en el servicio); después del cálculo estadístico se consideraron estadísticamente significativos los resultados cuyo valor de $p<0.05$. Los datos fueron sometidos a la prueba de normalidad Shapiro Willk, con los grupos poblacionales según la edad, género, estado civil y antigüedad en el servicio. Se tuvo en cuenta una significancia estadística inferencial de $\geqslant$ al 0.05 .

\section{Aspectos éticos}

Los principios y normas éticas en los que se sustenta el proyecto investigativo están vinculados a la Ley 1090 del 2006, Código Deontológico y Bioético del Psicólogo, donde se destaca la importancia de respetar y salvaguardar la información que brinde una persona o grupo al alcance del psicólogo. Se tramitó el permiso de la E.S.E para autorizar el acceso a la población y a sus instalaciones y la autorización a la divulgación general de los resultados.

\section{RESULTADOS}

Inicialmente se presentan los datos en dos momentos. En un primer momento, se exponen las características sociodemográficas de la población: edad, género, estado civil, área de servicio y antigüedad en la organización. En un segundo momento, se describe el nivel de satisfacción y sus diferencias significativas según el área de servicio y las características sociodemográficas. Para profundizar en el análisis se examinó el nivel de satisfacción de acuerdo con los factores psicosociales y ambientales percibidos por la población.

\section{Caracterización sociodemográfica}

En la Tabla 1 se evidencia que la muestra estuvo conformada en un $67 \%$ por mujeres, y en un $33 \%$ por hombres. En cuanto a la edad de los participantes, el 70\% son menores de 36 años y el $30 \%$ mayor de 37 años. El estado civil más destacado fue el soltero, con un $55 \%$; seguidamente la unión libre y/o casado (a) $40 \%$ y el $5 \%$ restante, corresponde a personas separadas. Con relación a la antigüedad en el área de servicio, se encontró el $65 \%$ con más de uno y menos de cinco años; el $23 \%$ con más de cinco años y el 12\% menos de 5 años desempeñando sus labores de enfermería en el hospital. 
Tabla 1. Datos sociodemográficos

\begin{tabular}{|l|l|l|}
\hline VARIABLE & ATRIBUTO & PORCENTAJE \\
\hline Género & Hombre & $33 \%$ \\
& Mujer & $67 \%$ \\
\hline Estado civil & Soltero & $55 \%$ \\
& Separado & $5 \%$ \\
& Casado & $40 \%$ \\
\hline Edad & $x_{e}<36$ años & $30 \%$ \\
& $x_{e}>37$ años & $70 \%$ \\
\hline Antigüedad & $x_{a}<1$ año & $12 \%$ \\
& 1 año $>x_{a}<5$ años & $65 \%$ \\
& $x_{a}>5$ años & $23 \%$ \\
\hline
\end{tabular}

$N=60$

Fuente: Elaboración de los autores

\section{Medias de nivel de satisfacción por área de servicio}

Al desagregar el nivel de satisfacción por área de servi- satisfacción con una media de 2,23 (DE 0,430), mientras cios se encontró que el personal auxiliar de enfermería el personal de la unidad crítica de neonatos presenta que pertenece al área de urgencias presenta mayor una media de 1,67 (DE 0,547).

Tabla 2. Rangos de satisfacción

\begin{tabular}{|l|l|l|l|}
\hline SERVICIO & MEDIA & N & DE \\
\hline Urgencias & 2,23 & 30 & 0,430 \\
\hline Neonatos & 1,67 & 30 & 0,547 \\
\hline Total & 1,95 & 60 & 0,565 \\
\hline
\end{tabular}

Fuente: Elaboración de los autores 
Nivel de satisfacción según variables sociodemográficas Al diferenciar el nivel de satisfacción por grupos (Tabla 3), no se encontraron diferencias significativas en los grupos según la edad, el género, el estado civil y la antigüedad del servicio de $p<0,05$. Mientras el nivel de satisfacción del personal asistencial varía según el área donde se presta el servicio, se encontraron diferencias significativas $p<0,001$.

Tabla 3. Diferencias significativas nivel satisfacción vs. datos sociodemográficos

\begin{tabular}{|c|c|c|c|c|c|}
\hline \multicolumn{2}{|c|}{ DATOS SOCIODEMOGRÁFICOS } & MEDIA & DE & F & SIGNIFICANCIA \\
\hline Género & $\begin{array}{l}\text { Hombre } \\
\text { Mujer }\end{array}$ & $\begin{array}{l}85,35 \\
79,82\end{array}$ & $\begin{array}{l}9,21 \\
10,50\end{array}$ & 3,990 & 0,050 \\
\hline Estado civil & $\begin{array}{l}\text { Soltero } \\
\text { Separado } \\
\text { Unión libre }\end{array}$ & $\begin{array}{l}79,15 \\
79,00 \\
85,45\end{array}$ & $\begin{array}{l}10,22 \\
9,64 \\
9,81\end{array}$ & 2,852 & 0,066 \\
\hline Edad & $\begin{array}{l}x_{e}<36 \text { años } \\
x_{e}>37 \text { años }\end{array}$ & $\begin{array}{l}80,42 \\
84,55\end{array}$ & $\begin{array}{l}9,75 \\
11,39\end{array}$ & 2,038 & 0,159 \\
\hline $\begin{array}{l}\text { Antigüedad en el } \\
\text { servicio }\end{array}$ & $\begin{array}{l}x_{a}<1 \text { año } \\
1 \text { año }>x_{a}<5 \text { años } \\
x_{a}>5 \text { años }\end{array}$ & $\begin{array}{l}85,14 \\
82,56 \\
77,42\end{array}$ & $\begin{array}{l}10,07 \\
9,45 \\
12,25\end{array}$ & 1,758 & 0,182 \\
\hline Área de Servicio & $\begin{array}{l}\text { Urgencias } \\
\text { Neonatos }\end{array}$ & $\begin{array}{l}87,96 \\
75,36\end{array}$ & $\begin{array}{l}7,66 \\
8,78\end{array}$ & 35,039 & $0,00 * *$ \\
\hline
\end{tabular}

** $p<0,001$

Fuente: Elaboración de los autores 9 factores considerando diferencias por el área donde se desempeñan los auxiliares de enfermería, se hacen evidentes las diferencias significativas $(p<0,001)$ en el nivel de satisfacción respecto a los factores: satisfacción en el trabajo, relaciones con jefes y monotonía.
Ahora bien, al analizar el nivel de SL por cada uno de los 
Tabla 4. Diferencias significativas según factores

\begin{tabular}{|c|c|c|c|c|c|}
\hline \multicolumn{2}{|l|}{ FACTORES } & \multirow{2}{*}{$\begin{array}{r}\text { MEDIA } \\
17,76 \\
14,40\end{array}$} & \multirow{2}{*}{$\begin{array}{l}\mathrm{DE} \\
1,99 \\
2,31\end{array}$} & \multirow{2}{*}{$\begin{array}{l}F \\
36,446\end{array}$} & \multirow{2}{*}{$\begin{array}{l}\text { SIGNIFICANCIA } \\
0,000^{*}\end{array}$} \\
\hline Satisfacción trabajo & $\begin{array}{l}\text { Urgencias } \\
\text { Neonatos }\end{array}$ & & & & \\
\hline $\begin{array}{l}\text { Competencia } \\
\text { profesional }\end{array}$ & $\begin{array}{l}\text { Urgencias } \\
\text { Neonatos }\end{array}$ & $\begin{array}{l}12,73 \\
11,23\end{array}$ & $\begin{array}{l}2,24 \\
2,28\end{array}$ & 6,586 & 0,013 \\
\hline $\begin{array}{l}\text { Promoción } \\
\text { profesional }\end{array}$ & $\begin{array}{l}\text { Urgencias } \\
\text { Neonatos }\end{array}$ & $\begin{array}{l}8,53 \\
7,83\end{array}$ & $\begin{array}{l}1,61 \\
2,18\end{array}$ & 1,995 & 0,163 \\
\hline Tensión trabajo & $\begin{array}{l}\text { Urgencias } \\
\text { Neonatos }\end{array}$ & $\begin{array}{l}16,36 \\
14,80\end{array}$ & $\begin{array}{l}2,85 \\
2,83\end{array}$ & 4,546 & 0,037 \\
\hline $\begin{array}{l}\text { Promoción } \\
\text { profesional }\end{array}$ & $\begin{array}{l}\text { Urgencias } \\
\text { Neonatos }\end{array}$ & $\begin{array}{l}8,53 \\
7,83\end{array}$ & $\begin{array}{l}1,61 \\
2,18\end{array}$ & 1,995 & 0,163 \\
\hline Relaciones jefes & $\begin{array}{l}\text { Urgencias } \\
\text { Neonatos }\end{array}$ & $\begin{array}{l}7,30 \\
5,60\end{array}$ & $\begin{array}{l}1,23 \\
2,02\end{array}$ & 15,378 & $0,000^{*}$ \\
\hline $\begin{array}{l}\text { Relaciones } \\
\text { compañeros }\end{array}$ & $\begin{array}{l}\text { Urgencias } \\
\text { Neonatos }\end{array}$ & $\begin{array}{l}4,06 \\
4,06\end{array}$ & $\begin{array}{l}0,69 \\
0,69\end{array}$ & 0,000 & 1,000 \\
\hline $\begin{array}{l}\text { Características } \\
\text { extrínsecas }\end{array}$ & $\begin{array}{l}\text { Urgencias } \\
\text { Neonatos }\end{array}$ & $\begin{array}{l}6,06 \\
5,20\end{array}$ & $\begin{array}{l}1,68 \\
1,29\end{array}$ & 5,001 & 0,029 \\
\hline Monotonía & $\begin{array}{l}\text { Urgencias } \\
\text { Neonatos }\end{array}$ & $\begin{array}{l}8,66 \\
6,63\end{array}$ & $\begin{array}{l}1,24 \\
1,79\end{array}$ & 26,134 & $0,000 *$ \\
\hline Presión trabajo & $\begin{array}{l}\text { Urgencias } \\
\text { Neonatos }\end{array}$ & $\begin{array}{l}6,46 \\
5,60\end{array}$ & $\begin{array}{l}2,43 \\
1,99\end{array}$ & 2,280 & 0,137 \\
\hline Índice satisfacción & $\begin{array}{l}\text { Urgencias } \\
\text { Neonatos }\end{array}$ & $\begin{array}{l}87,96 \\
75,36\end{array}$ & $\begin{array}{l}7,66 \\
8,78\end{array}$ & 35,039 & $0,000 *$ \\
\hline
\end{tabular}

$* p<0.001$

Fuente: Elaboración de los autores 


\section{DISCUSIÓN}

Este artículo exploró los niveles de satisfacción de un grupo de auxiliares de enfermería en una institución pública prestadora de servicios de salud de tercer nivel en la ciudad de Popayán, Colombia. Contexto y temática poco explorada a nivel investigativo. Los resultados señalan tendencia de nivel de satisfacción medio en el $68 \%$ de las personas estudiadas. Datos consistentes con los hallazgos de Cifuentes-Rodríguez y ManriqueAbril (2015), Holguín y Contreras (2020), Orcasita y Ovalle (2019) y Plascencia et al. (2016). La ausencia de mejores niveles de $S L$, son una señal de alerta para el sistema de salud. A las múltiples decisiones políticas, sociales, económicas que han venido amenazando el bienestar de los profesionales o usuarios de las instituciones de salud se abona la emergencia sanitaria. Se requieren acciones coordinadas basadas en evidencia empírica que tranversalicen de manera integral y con el mismo nivel de importancia la necesidad de ofertar servicios con altos estándares de calidad y bienestar del personal sanitario.

Con un mayor nivel de exploración estadística, no se encontraron diferencias significativas entre el nivel de satisfacción con variables sociodemográficas ledad, género, estado civil y antigüedad en el serviciol, tales resultados contradicen los hallazgos de VázquezColunga et al. (2021), quienes sugieren intervenir sobre estas variables para mejorar la salud laboral de los trabajadores. Vale aclarar que, pese a no encontrarse diferencias significativas desagregadas por género, los resultados si presentan distinciones en el nivel de satisfacción en contra de las mujeres; el mercado laboral no les ofrece oportunidades justas y equitativas. Especialmente las enfermeras presentan un gran número de funciones y responsabilidades que no compensan con el salario evidentemente inferior comparado al de personas que tienen oportunidades de profesionalización como los médicos, que a propósito, son en su mayoría hombres (Ministerio de Salud, 2018).

También se encontraron diferencias significativas en el nivel de satisfacción con el factor relación jefes en cuanto a los dos servicios evaluados (Urgencias y Neonatos); es decir, la existencia de un bajo nivel de satisfacción en el área de Neonatos. Al respecto, Cook et al. (2021) y García et al. (2021) resaltan la importancia de mantener adecuadas relaciones interpersonales con compañeros y el buen liderazgo para mantener una positiva competencia profesional y satisfacción por el trabajo. Un alto grado de reconocimiento proporcionado por los jefes impacta significativamente en la SL.

Respecto al factor monotonía la investigación señaló diferencias significativas en los dos servicios encuestados. Se considera que un trabajo dinámico y buena comunicación elevan los niveles de SL. La monotonía es considerada un factor de riesgo para la satisfacción (Herrera y Manrique, 2019) dado que afecta a los profesionales en la toma de decisiones, prácticas rutinarias de trabajo y pocas posibilidades para la creación de nuevas ideas.

Un aspecto importante para resaltar en los resultados de la presente investigación se refiere al hecho de encontrar diferencias estadísticamente significativas para las áreas de servicio. Esto pone en evidencia la importancia del contenido de la tarea y el puesto de trabajo en los niveles de SL de los trabajadores en el área de la salud (Rivera-Rojas et al. 2021). Los ambientes y condiciones laborales impactan en la percepción de la SL (Orejuela, 2014; Bravo et al., 2017). 
De acuerdo con los hallazgos encontrados las organizaciones deben contemplar la posibilidad de generar cambios positivos en la confianza, el apoyo y la flexibilidad, así como tomar acciones para proteger la salud física y emocional de los trabajadores (Oliva -Yarlaque y Chávarry-Ysla, 2021). Por otra parte, la relación ser humano - trabajo implica un vínculo de reciprocidad. Para García y Forero (2014) esto se da cuando la organización brinda oportunidades para satisfacer las necesidades y proyectos de los trabajadores, cuando estos manifiestan el compromiso necesario para cumplir las metas organizacionales.

\section{CONCLUSIONES}

Las organizaciones adoptan iniciativas y diferentes estrategias para incrementar los niveles de satisfacción en las personas. Sin embargo, la tarea en pro de la dignidad del trabajo en las organizaciones, aún se encuentra en deuda. Las Ciencias Sociales deben amplificar las voces de las personas que en lo laboral presentan situaciones de exclusión y marginalidad.

En términos generales, las (los) auxiliares de enfermería de la muestra estudiada se ubican en un rango medio de satisfacción en los factores evaluados, lo que amerita observación y estrategias de intervención que coadyuven a elevar el nivel de satisfacción en trabajadores.

Pese a que las dos áreas de servicios evaluados pertenecen a la misma organización, al compararlos, se encontraron diferencias significativas en la percepción de SL, particularmente en los factores: satisfacción por el trabajo, relación jefes y monotonía. Se destaca que el liderazgo constituye un papel fundamental para la generación de confianza, comodidad, redes de apoyo emocional, fortalecimiento de identidad y de la autoestima
Es importante subrayar que, respecto a la satisfacción según el género, a pesar de no encontrarse diferencias significativas las mujeres son quienes expresan mayor insatisfacción laboral, por lo que es pertinente desarrollar futuras líneas de investigación orientadas a indagar la temática en función del género. Igualmente, continuar planteando investigaciones referidas a la SLen el personal de salud, es imperativo, debido a la connotación psicosocial y de sentido que tiene el trabajo en la vida de las personas; además, de las dinámicas que suscitan en este personal a razón de las políticas y reformas que los someten. La generación de nuevos conocimientos y problematizaciones alrededor del trabajo redundará en soluciones para enriquecer y dignificar el rol del auxiliar de enfermería.

\section{DECLARACIÓN DE CONFLICTO DE INTERÉS}

Los autores no declaran conflicto de interés. 


\section{REFERENCIAS}

Ansoleaga, E. y Ahumada, M. (2020). Factores de riesgo y protección para la salud mental de trabajadoras/es de salud pública postcatástrofes. Praxis Psy 21(34), 40-48.

Aranaz, J. M. (1988). Cuestionario Font Roja. Un instrumento de medida de la satisfacción en el medio hospitalario. Todo hospital (52), 63-68.

Ato, M., López, J. J., \& Benavente, A. (2013). Un sistema de clasificación de los diseños de investigación en psicología. Anales de Psicología, 29(3), 10381059. doi:10.6018/analesps.29.3.178511

Avendaño, W., Luna, H. y Rueda, G. (2021). Satisfacción laboral de los docentes: un análisis desde los factores extrínsecos e intrínsecos. Revista venezolana de Gerencia, 26 (5), 190-201. https://doi.org/10.52080/rvgluz.26.e5.13

Blanch, J. M. (2014). Calidad de vida laboral en hospitales y universidades mercantilizados. Papeles del Psicólogo, 35(1), 40-47.

Bravo, I., Díaz, A., Navarrete, C., Pérez, M., Cuevas, C., Nova, C., \& Albornoz, M. (2017). Valoración de la influencia de un programa de acompañamiento directivo sobre la percepción y satisfacción laboral de docentes y directivos. Universitas Psychologica, 16(2), 1-10. https://doi.org/10.11144/Javeriana.upsy16-2.vipa

Cardoza, M., Hidalgo, L., Peña, J. y Torres, C. (2019). Satisfacción con la vida y satisfacción laboral. Revista de investigación y cultura, 8 (1), 75-84. https://www. redalyc.org/articulo.oa?id=521758809021

Carlos, M. (2020). Síndrome de burnout y satisfacción laboral en el profesional de enfermería del Hospital Belén Lambayeque. Revista epistemia, 4 (1), 27-38. http://revistas. uss.edu.pe/index.php/EPT/article/view/1306/1231

Chiang, M y Ojeda, J. (2013). Estudio de la relación entre satisfacción laboral y el desempeño de los trabajadores de las ferias libres. Contaduría y administración, 58 (2), 39-60. DOI: 10.1016/S0186-1042(13)71209-9

Cifuentes-Rodríguez, J. E. y Manrique-Abril, F. G. (2014) Satisfacción laboral en enfermería en una institución de salud de cuarto nivel de atención: Bogotá,

Colombia. Avances en Enfermería., 32 (2), 217-227.

Cook, L.J., Hassem, T., Laher, S., Variava, T., \& Schutte, E. (2021). Mental health experiences of healthcare professionals during COVID-19. SA Journal of Industrial Psychology/SA Tydskrif vir Bedryfsielkunde, 47 (0), 1-10. https://doi.org/10.4102/sajip.v47i0.1865

Da Penha, S., Da silva, R., Minimel, A., Moraes, T., y Silveira, T. (2019). Calidad de vida en el trabajo y perfil demográfico-laboral de la enfermería en unidad de urgencias. Revista Enfermería global, 18 (55), 510-523. doi: 10.6018/eglobal.18.3.340861

Dantas, M., Cunha, E., Dantas, M., Santos, P. y Tavares, C. (2020). Angustia Emocional de las Enfermeras en el contexto hospitalario que enfrenta la pandemia de COVID-19. Research, Society and development, 9 (8), 1-21. doi: http://dx.doi.org/10.33448/rsd-v9i8.5121

De la Iglesia, G., Lupano, M. y Castro, A. (2019). Modelo de Personalidad Positiva: su asociación al funcionamiento óptimo en trabajadores activos. Revista de psicología, 37 (2), 425-229. https://revistas. pucp.edu.pe/index. php/psicologia/article/view/20979/20664

Duche, A. y Rivera, G. (2019). Satisfacción laboral y felicidad en enfermeras peruanas. Enfermería global, 18 (2), 353373. https://doi.org/10.6018/eglobal.18.2.334741

Gálvez-Hernando, G., Selva-Olid, C., \& Blanch, J. M. (2019). Violencia ocupacional en un hospital manifestada a través de reclamaciones. Athenea Digital, 19(2), e2247. https://doi.org/10.5565/rev/athenea.2247

García, L., Giraldo, D., Aguirre-Loaiza, H., Nuñoz, C. y QuirozGonzalez, E. (2021). Calidad de vida laboral y autoeficacia profesional en docentes de educación superior. Praxis, 17 (1), 85-98. http://revistas.unimagdalena. edu.co/index.php/praxis/article/view/3539/2987

García, M. y Forero, C. (2014). Motivación y satisfacción laboral como determinantes asociados al cambio organizacional. En J. Orejuela (Ed.), Psicología de las organizaciones y del trabajo: apuestas de investigación (pp. 327-349). Editorial Bonaventuriana. 
Griffin, R., Phillips, J. y Gully, S. (2017). Comportamiento organizacional: Administración de personas y

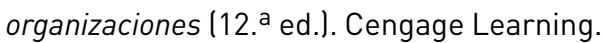

Hernández, R., Fernández, C., \& Baptista, M. (2014). Metodología de la investigación. Mc Graw Hill.

Herrera, G., y Manrique, F. (2019). Satisfacción laboral de enfermería. Diferencias de ámbitos en atención primaria en salud en cuidado intensivo: Universidad Nacional de Colombia. doi:10.21676/2389783X.2943

Holguín, Y. y Contreras, C. (2020). Satisfacción laboral del personal en el Servicio de Nefrología del Hospital Guillermo Kaelin de la Fuente (Villa María) en 2019. Horizonte médico (Lima), 20 (2), 12-38. https://doi.org/10.24265/horizmed.2020.v20n2.09

Li, Y., Huang, H. \& Chen, Yi. (2020). Organizational climate, job satisfaction, and turnover in voluntary child welfare workers. Children and Youth Services Review, 119, 10-56. https://doi.org/10.1016/j.childyouth.2020.105640

López-Roldán, P., y Fachelli, S. (2016). Análisis de varianza. En P. López-Roldán, y S. Fachelli, Metodología de la Investigación Social Cuantitativa. Universitat Autònoma de Barcelona. Obtenido de https://ddd.uab.cat/record/163568

Madero-Gómez, S. (2020). La satisfacción laboral como efecto moderador entre las prácticas organizacionales de recursos humanos y las líneas estratégicas de responsabilidad social corporativa en México. Estudios gerenciales, 36 (157), 391-401. https://doi.org/10.18046/j.estger.2020.157.3790

Manrique-Abril, F., Herrera-Amaya, G. M., \& Méndez-Fandiño, Y. R. (2019). Validez y fiabilidad en Colombia del Font Roja. Hacia la Promoción de la Salud, 24(2), 46-59. DOI: 10.17151/hpsal.2019.24.2.5

Ministerio de Salud. (2018). Política Nacional de Talento Humano en Salud. Bogotá, DC. Recuperado de https://bit.ly/3mXe3H9

Newstrom, J. (2011). Comportamiento humano en el trabajo (13a ed.). McGraw-Hill.

Oliva-Yarlaque, Y. y Chávarry - Ysla, P. (2021). Impacto Emocional en el Profesional de Enfermería Durante la Pandemia. Revista Recién, 10 (2), 186-193. https://revista.cep. org.pe/index.php/RECIEN/article/view/85
Orcasita, A. y Ovalle, L. (2019). Condiciones laborales del personal de Enfermería en una clínica de alta complejidad en Valledupar. Biociencias, 14(1). doi: 0.18041/2390-0512/biociencias.1.5334

Orejuela, J. (2014). La identidad de la evaluación subjetiva del trabajo: de la satisfacción al sufrimiento laboral. En J. Orejuela (Ed.), Psicología de las organizaciones y del trabajo: apuestas de investigación (págs. 163-189). Editorial Bonaventuriana.

Pedraza, N. (2018). El clima y la satisfacción laboral del capital humano: factores diferenciados en organizaciones públicas y privadas. Innovar, 30 (76), 9-24. https:// doi.org/10.15446/innovar.v30n76.85191.

Plascencia, C., Pozos, R., Preciado, S., \& Vázquez, G. (2016). Satisfacción Laboral del personal de enfermería de una institución pública de Jalisco, México. Revista Cubana de Salud y Trabajo. https://www.medigraphic. com/pdfs/revcubsaltra/cst-2016/cst162g.pdf

Pujol-Cols, L. J. y Dabos, G. E. (2018). Satisfacción laboral: una revisión de la literatura acerca de sus principales determinantes. Estudios Gerenciales, 34 (146), 3-18. https://doi.org/10.18046/j.estger.2018.146.2809

Pujol-Cols, L. (2019). Core Self-Evaluations, Perceived Job Characteristics and Job Satisfaction: Evidence from Two Independent Samples of Highly Skilled Argentinian Workers. Revista colombiana de Psicología, 28 (1), 131 146. doi: https://doi.org/10.15446/rcp.v28n1.70420

Pujol-Cols, L. y Foutel, M. (2019). Satisfacción laboral y salud: Un análisis de efectos directos e indirectos en gerentes argentinos. Cuadernos de administración, 32 (59), 1-22. https://doi.org/10.11144/Javeriana.cao32-59.slsaed

Rivera-Rojas, F., Ceballos-Vázques,P. y González-Palacios, Y. (2020). Riesgos psicosociales y satisfacción laboral: una relación significativa para los trabajadores de oncología. Aquichan, 21 (2), 1-11. DOI: https://doi.org/10.5294/aqui.2021.21.1.4

Robbins, S. y Judge, T. (2017). Comportamiento organizacional. (17. a ed.). Pearson Educación

Ruiz, J. K. (2019). Algunos determinantes de la satisfacción laboral en Colombia. Economía \& Región, 7(2), 91-118. 
Salessi, S. y Omar, A. (2018). Tríada oscura de personalidad, satisfacción laboral y cinismo organizacional: un modelo estructural. Universitas Psychologica, 17(3), 1-12. https://doi.org/10.11144/Javeriana.upsy17-3.tops

Spector, P. (1997). Job Satisfaction: Applications, Assessment, Causes and Consequences. SAGE.

Troya, C., Padilla, M., Camacho, L. y Benavidez, L. (2020). Evaluación de la satisfacción laboral en un grupo de enfermeras de cuatro ciudades del Ecuador en el mes de enero de 2020. Práctica Familiar Rural, 5 (1), 1-12. doi: https://doi.org/10.23936/pfr.v5i1.145

Vargas, T., Vizzuett, V., Montiel, E., Becerra, L. y Villegaz, E. (2018). La satisfacción laboral y su influencia en la productividad. Teuken Bidikay, 9 (13), 129-153. https://revistas.elpoli. edu.co/index.php/teu/article/view/1444/1094

Vázquez-Colunga, J., Vásquez-Juaréz, C., Ángel-González, M., Valadez-García, J., de la Roca- Chiapas, J., Colunga-Rodriguez, C. \& Colunga-Rodriguez, B. (2021). Sociodemographic characterization of job satisfaction in hospital staff. Scielo preprints, 1-14. https://doi.org/10.1590/SciELOPreprints.1823

Veliz- Burgos, A. A., Dörner-Paris, A. P., Soto- Salcedo, A., Reyes Lobos, J. L., \& Ganga Contreras, F. (2018). Inteligencia emocional y bienestar psicológico en profesionales de enfermería del sur de Chile. Medisur 16(2). 259-266. 